九州大学学術情報リポジトリ

Kyushu University Institutional Repository

Spectral Analysis of Field Potential Recordings by Deep Brain Stimulation Electrode for Localization of Subthalamic Nucleus in Patients with Parkinson' s Disease

Miyagi, Yasushi

Digital Medicine Initiative, Kyushu University : Associate Professor

http://hdl. handle. net/2324/16988

出版情報: Stereotactic and Functional Neurosurgery. 87, pp.211-218，2009. S. Karger AG, Basel (Switzer land) バージョン :

権利関係 : 


\title{
Spectral Analysis of Field Potential Recordings by Deep Brain Stimulation Electrode for Localization of Subthalamic Nucleus in Patients with Parkinson's Disease
}

\author{
Yasushi Miyagi ${ }^{a}$ b Tsuyoshi Okamoto $^{a}$ Takato Morioka $^{b}$ Shozo Tobimatsu ${ }^{c}$ \\ Yoshitaka Nakanishi $^{a}$ Kazuyuki Aiharad Kimiaki Hashiguchi ${ }^{b}$ \\ Nobuya Murakami ${ }^{e}$ Fumiaki Yoshida ${ }^{b}$ Kazuhiro Samura $^{b}$ Shinji Nagata ${ }^{b}$ \\ Tomio Sasaki ${ }^{b}$ \\ ${ }^{\mathrm{a}}$ Digital Medicine Initiative and Departments of ${ }^{\mathrm{b}}$ Neurosurgery and ${ }^{\mathrm{C} C l i n i c a l}$ Neurophysiology, \\ Graduate School of Medical Sciences, Kyushu University, ${ }^{d}$ Aihara Complexity Modeling Project, ERATO, JST, and \\ e Department of Neurosurgery, Kaizuka Hospital, Fukuoka, Japan
}

\section{Key Words}

Oscillatory synchrony $\cdot$ Subthalamic nucleus $\cdot$ Local field potential $\cdot$ Spectral density analysis $\cdot \beta$-Activity $\cdot$ Deep brain stimulation $\cdot$ Parkinson's disease

\begin{abstract}
Aims: Spectral analysis of local field potential (LFP) recorded by deep brain stimulation (DBS) electrode around the subthalamic nucleus (STN) in patients with Parkinson's disease was performed. Methods: The borders of the STN were determined by microelectrode recording. The most eligible trajectory for the sensorimotor area of the STN was used for LFP recording while advancing the DBS electrode. Results: The low-frequency LFP power ( $\theta$ - to $\beta$-band) increased from a few millimeters above the dorsal border of the STN defined by microelectrode recording; however, the low-frequency power kept the same level beyond the ventral border of the STN. Only high $\beta$-power showed close correlation to the dor-
\end{abstract}

sal and ventral borders of the STN. Conclusions: A spectral power analysis of LFP recording by DBS electrode helps with the final confirmation of the dorsal and ventral borders of the STN of Parkinson's disease in DBS implantation surgery.

Copyright $\odot 2009$ S. Karger AG, Basel

\section{Introduction}

In Parkinson's disease (PD), it is postulated that dopamine depletion in the striatum leads to an oscillatory synchrony in low-frequency ( $\beta$-band) neuronal discharges of the basal ganglia [1]. Such oscillatory synchrony is considered to induce the antikinetic effect on the interaction between the motor cortex and basal ganglia in the 'off' state of PD, but when the patient's motor function is turned to the 'on' state by levodopa treatment, the $\beta$-band oscillation disappears or changes into $\gamma$-band oscillation [2].

\section{KARGER}

Fax +41613061234 E-Mail karger@karger.ch www.karger.com
(C) 2009 S. Karger AG, Basel

$1011-6125 / 09 / 0874-0211 \$ 26.00 / 0$

Accessible online at:

www.karger.com/sfn
Yasushi Miyagi, MD, $\mathrm{PhD}$

Division of Digital Patient, Digital Medicine Initiative, Kyushu University

3-1-1 Maidashi, Higashi-ku, Fukuoka, 812-8582 (Japan)

Tel. +81 92642 6693, Fax +81926426693

E-Mail yamiyagi@digital.med.kyushu-u.ac.jp 
In some studies on local field potential (LFP) in the basal ganglia, a low-impedance microelectrode was used during stereotactic neurosurgery [3-5]. However, the LFPs can be recorded using a deep brain stimulation (DBS) electrode during surgery [6-8] or during the trial period after implantation with an externalized cable $[7$, 9]. Chen et al. [6] reported that the subthalamic nucleus (STN) can be physiologically localized by DBS electrode alone, without performing microelectrode recording (MER). In their study a DBS electrode was inserted by a 2-mm step. When the contact got into the STN, a spectral change was observed. However, the details of the border of the STN were not identifiable with the 2-mm step insertion. Since the depth of the dorsal and ventral borders defines the physiological width of the STN, which is considered a major determinant of the eligible trajectory for the implantation of a DBS electrode $[10,11]$, the detailed characteristics of LFP around the dorsal and ventral borders provide crucial information if MER is not performed. The purpose of this study is to characterize the LFP changes around the STN borders, and to identify the dorsal and ventral STN border by the change in LFP from the DBS electrode.

\section{Materials and Methods}

\section{Patients}

Nine STNs from 7 patients with idiopathic PD (2 males and 5 females, with a mean age of $65 \pm 5.3$ years ranging from 50 to 71 ) were included for the LFP recording during DBS surgery. All patients had disabling motor fluctuations and/or levodopa-induced dyskinesia refractory to the adjustment of antiparkinsonian medication. The mean disease duration was $14 \pm 8.5$ years, and the mean Hoehn-Yahr stage was $3.9 \pm 1.1$ in the 'off' state and $2.7 \pm$ 0.8 in the 'on' state. The patients were treated with levodopa and dopamine agonists; the daily dose of levodopa was $586 \pm 306$ $\mathrm{mg} /$ day (total levodopa equivalent dose $=884 \pm 458 \mathrm{mg} /$ day). In 5 patients, only unilateral STN was explored for LFP recording because of limited operation time.

\section{Surgical Procedure}

All patients gave their informed consent for DBS implantation surgery and related data acquisition. Surgeries were performed in the 'off' state after an 18-hour discontinuation of antiparkinsonian medication. After a stereotactic head frame (Leksell model G, Elekta) had been affixed to the patient's head under local anesthesia, the patient underwent preoperative magnetic resonance (MR) imaging and helical computerized tomography (CT). The axial images were transferred to the workstation computer in the operating room in a DICOM format. The data were imported into the computer software SurgiPlan ${ }^{\mathrm{TM}}$ (Elekta, Sweden). After both 3-dimensional images reconstructed from MR and CT had been exactly matched, as confirmed by the image fusion tool, the coor- dinates of the anterior and posterior commissures (AC and PC) were directly calculated by the software. The theoretical target was placed 11-12 $\mathrm{mm}$ lateral to the AC-PC line, $3 \mathrm{~mm}$ posterior and $5 \mathrm{~mm}$ ventral to the midcommissural point. The laterality of the theoretical target from the AC-PC line was also individually modified, as $3 \mathrm{~mm}$ lateral to the lateral border of the red nucleus visualized on a $\mathrm{T}_{2}$-weighted coronal image in each case. The coordinates were also calculated separately by manual drawing on the X-ray film, and a consensus was obtained with the results from the 'functional target' tool of SurgiPlan. The entry point (site of burr hole) was placed around the coronal suture and 3-4 cm lateral to the midline; the final trajectory was planned to avoid the cortical sulci and lateral ventricle. The patient's head with head frame was secured to the operating table, and the patient was postured in a supine position to prevent a brain shift during the burr hole surgery [12].

\section{Multitrack Microrecording}

A semicircular skin incision and burr hole were made at the entry point. Immediately after the dura mater had been opened, the burr hole was covered with fibrin glue to prevent the outflow of cerebrospinal fluid during surgery. Four cannulae for multitrack MER (Array electrode insertion tube ${ }^{\circledR}$, Medtronic Inc., Minneapolis, Minn., USA) were inserted into the cortex. The STN was physiologically localized by 4 simultaneously inserted tracks of microelectrode (microTargeting Electrode ${ }^{\circledR}$, Medtronic) [13]. MER was started $15 \mathrm{~mm}$ above the theoretical target and conducted by a manual microdrive device. Extracellular potentials were sampled at a rate of $24 \mathrm{kHz}$ by a surgical monitoring system (Leadpoint ${ }^{\circledR}$, Medtronic) and the data were collected every $10 \mathrm{~s}$ at each depth. The discharge pattern of the neurons of the subthalamus and substantia nigra pars reticulata $(\mathrm{SNr})$ could be identified as follows: characteristic neuronal discharges of the STN were identified by the robust increase in background activity with multiple units with a relatively large amplitude and nontonic irregular discharge pattern with a firing rate of around $30-60 \mathrm{~Hz}$. As the electrode went beyond the STN, the background activity substantially decreased and the SNr cells with high-frequency and tonic discharges appeared. The boundary between STN and SNr was not always clear; therefore, the ventral border of the STN was defined by offline analysis. The sensorimotor area of the STN was distinguished by the modification of neuronal discharges in response to active and passive joint movement of contralateral limbs. The track recording the sensorimotor response of the STN and a width of STN larger than $4 \mathrm{~mm}$ was regarded as the eligible trajectory for DBS electrode implantation $[10,11,14]$.

\section{LFP Recording by DBS Electrode}

After the dorsal and ventral borders of the STN had been identified by MER, the DBS electrode (model 3389, Medtronic) with 4 platinum-iridium cylindrical surfaces $(1.27 \mathrm{~mm}$ diameter with $1.5 \mathrm{~mm}$ length) and $0.5-\mathrm{mm}$ intervals was introduced to the microdrive device. The DBS electrode was mounted to keep a spatial relationship so that the center between the 2 lowermost bipolar contacts coincided with the depth of the microelectrode tip. LFPs were recorded by use of the LeadPoint system, too. The sensitivity was adjusted to $0.1 \mathrm{mV}$ per dot in the display and the sampling rate was $24 \mathrm{kHz}$. Using the lowermost 2 contacts (contact 0 and contact 1), the LFP was recorded from $15 \mathrm{~mm}$ above the theoretical target down to $5 \mathrm{~mm}$ beyond the theoretical target in a 1- $\mathrm{mm}$ 

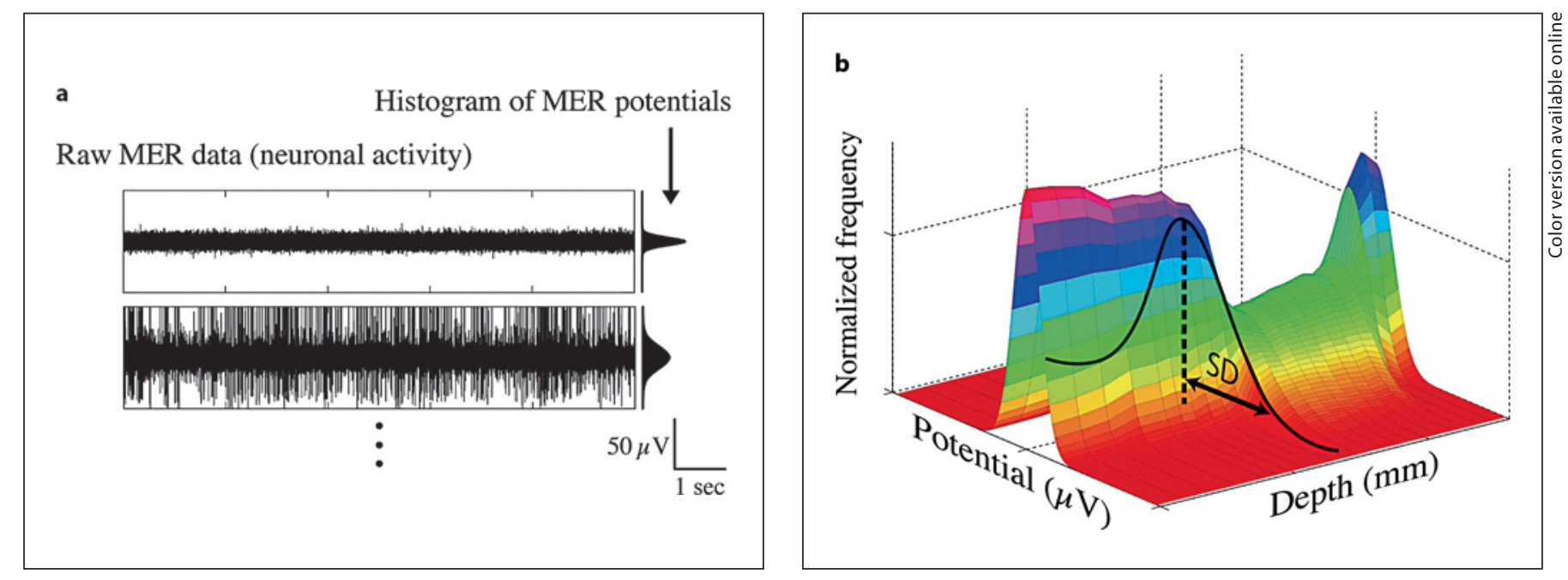

Fig. 1. Method of offline analysis of MER in determining the electrophysiological border of the STN. a The SD of 240,000 data points ( $24 \mathrm{kHz}$ for $10 \mathrm{~s}$ ) of MER potential was regarded as a background activity of each depth. $\mathbf{b}$ The relationship between the histogram of MER potential and the depth of each microelectrode. c The SD of MER potentials at each depth was expressed as a percentage of maximum SD in each patient and the depths where percent SD increased up to $50 \%$ and subsequently decreased down to $50 \%$ were defined as dorsal and ventral borders of the STN, respectively.

step with filtration of $1-50 \mathrm{~Hz}$. The patients were kept at rest without any voluntary movement during LFP recording because the degree of synchronization of STN neurons in the $\beta$-band is dependent on patients' movement $[9,15]$. The DBS electrode was not advanced further than $5 \mathrm{~mm}$ from theoretical target.

\section{Macrostimulation and Implantation}

The DBS electrode was withdrawn to the depth where the lowermost 2 contacts were located within the STN as determined by MER, and the electrode location was confirmed by intraoperative roentgenogram. The parameters used for macrostimulation by DBS electrode were as follows: bipolar stimulation with contact 1 (dorsal half of the STN) as cathode and contact 3 as anode, pulse width $60 \mu \mathrm{s}$, frequency $130 \mathrm{~Hz}$. The muscle rigidity was significantly decreased by insertion of the DBS electrode even in the absence of electrical stimulation (microlesioning effect). In all cases, the amplitude could be gradually increased up to $5.0 \mathrm{~V}$ without the generation of adverse effects, such as dysarthria, conjugate deviation or dystonic muscle contraction. After getting the optimal therapeutic effect by the refinement of the electrode posi-

tion, the electrode was fixed with the Medtronic burr hole cap. The final position of the electrode tip was consistently ascertained by intraoperative roentgenogram. The same procedure was then repeated for the other side. Finally, the patients underwent the implantation of an extension cable and an internal pulse generator under general anesthesia on the same day.

\section{Frequency Analysis}

Since the high background neuronal activity reflects both high-frequency spikes and high neuronal density, the changes in the histogram of MER potentials are considered to correlate with both the neuronal density of the STN and the spiking activity of neurons. Therefore, the standard deviation (SD) of 240,000 data points ( $24 \mathrm{kHz}$ for $10 \mathrm{~s}$ ) of MER potential was regarded as a background activity at each depth and used as an electrophysiological marker of STN (fig. 1a, b). The background activity began to increase a few millimeters above the estimated dorsal border before it turned into a steep increase in each trajectory. In order to avoid the vagueness of the STN border in this study, the SD of MER potentials at each depth was expressed as a percentage of the maxi- 
mum SD in each patient, and the depths where the SD increased up to $50 \%$ of maximum and subsequently decreased down to $50 \%$ of maximum were defined as the dorsal and ventral borders of STN, respectively (fig. 1c).

The power spectral density (PSD) was calculated by Fourier transform with Welch's modification [16] over the 10-second sequence of LFP which is segmented into 1-second sections overlaid with a Hamming window, each with a $50 \%$ overlap. In the spectral density analysis of LFP around the dorsal border of the STN, 9 data sets of MER and LFP records were aligned while assuming the depth of the dorsal border as $0 \mathrm{~mm}$. In case of LFP analysis around the ventral border of the STN, 9 data sets of MER and LFP records were realigned while assuming the depth of the ventral border as $0 \mathrm{~mm}$. The maximum power was obtained in each band of $\theta(4-7 \mathrm{~Hz}), \alpha(8-13 \mathrm{~Hz})$ and $\beta(14-35 \mathrm{~Hz})$. In particular, the $\beta$ band was further divided into low $\beta(14-20 \mathrm{~Hz})$ and high $\beta(21-35$ $\mathrm{Hz}$ ). The relationships between the DBS electrode depth to the dorsal and ventral borders of the STN determined by MER and the LFP power of each band recorded by DBS electrode were analyzed.

Statistical Analysis and Ethical Aspects

The LFP response recorded by DBS electrode is related to the level of activity of large groups of neurons. The PSD change of LFP with the depth does not always show the same trend as the background activity recorded by MER electrode. In order to test the significance of PSD increase and decrease of LFP with the location of the electrode, the correlation analysis between the normalized PSD and the depth of the electrode located between -2 and $2 \mathrm{~mm}$ around the ventral border or dorsal border of the STN, respectively, was performed. In this analysis, both the correlation coefficient $R$ and the $\mathrm{p}$ value for testing the hypothesis of no correlation were calculated in each band of $\theta, \alpha$ and $\beta$. If the $p$ value is less than 0.05 , then the correlation coefficient $R$ is significant. This study was approved by the Research Ethics Committee, Kyushu University (No. 20-16).

\section{Results}

The width of the STN determined by MER was $5.2 \pm$ $0.6 \mathrm{~mm}$ along the eligible track for DBS electrode implantation. Figure 2 shows the representative records of a 65year-old male patient indicating the dependency of PSD on the depth of the DBS electrode from dorsal to ventral of the STN through the trajectory, including the sensorimotor area detected by multitrack MER. The power of the low-frequency band clearly increased around the dorsal border of the STN at $-4 \mathrm{~mm}$ to the tentative target ( $0 \mathrm{~mm}$ depth) in figure 2 and low-frequency synchrony was kept at the same level beyond the ventral border of the STN; only the high $\beta$-power of LFP showed a clear decrease around the ventral border.

The summary of 10 LFP recordings is shown in figure 3, in which the depth-power relationships were aligned in reference to the dorsal (fig. 3b-e) and ventral (fig. 3g-j) borders, which are designated as $0 \mathrm{~mm}$ depth, respectively. Although the background activity in all MER data showed a steep increase around the dorsal border of the STN (fig. 3a), the LFP power of each low-frequency band began to increase from a few millimeters above the dorsal border. As a whole, the depth at which $\theta$-, $\alpha$ - and low $\beta$-powers reached almost half of the maximum power coincided with the dorsal border (depth 0 $\mathrm{mm}$ ); at the depth of $2 \mathrm{~mm}$ dorsal to the STN, however, only high $\beta$-power reached half of the maximum power $(R=0.48, \mathrm{p}<0.001$, fig. $3 \mathrm{e})$. As the DBS electrode advanced ventrally, the LFP power appeared to increase within the STN or remained the same, and when aligned by the ventral border of the STN, the LFP power of the $\theta$ - to low $\beta$-band did not show any significant change around the ventral border (depth $0 \mathrm{~mm}$ ). Only the high $\beta$-power showed a small but significant decline just at the ventral border $(R=-0.37, \mathrm{p}=0.01$, fig. $3 \mathrm{j})$.

\section{Discussion}

In this study, we performed an LFP recording from a DBS electrode to detect any change in spectral density of LFP specific to the borders of the STN along the trajectory of the DBS electrode. An approximate $50 \%$ increase from the baseline in the LFP power of low-frequency $(\theta-$ to low $\beta$-) bands correlated with the dorsal STN border. However, the LFP power of these bands did not show any significant change around the ventral border. Therefore, the increase in oscillatory activity in a low-frequency band is clear when the DBS electrode moves from Forel's field or the zona incerta into the dorsal STN, while the decrease in oscillatory activity is unclear around the ventral STN. These results suggest that low-frequency LFP power recording by DBS electrode can be used only for the detection of the dorsal border of the STN and the high $\beta$-power of LFP can help detect the ventral border of the STN.

Dopaminergic depletion in the striatum induces the $\beta$-band oscillatory synchrony of neuronal activity in the STN and globus pallidus internus, which contributes to the antikinetic effect on the basal ganglia in the 'off' state of PD [1]. The use of $\beta$-power of LFP is reasonable for the electrophysiological localization of the STN $[6,4]$. An LFP recording by a DBS electrode has several technical problems to be overcome. A simultaneous recording of LFP and neuronal discharge revealed that the mean $\beta$ power $(13-35 \mathrm{~Hz})$ was greater in the dorsolateral than in the ventromedial STN [3], and a majority of $\beta$-oscillatory 


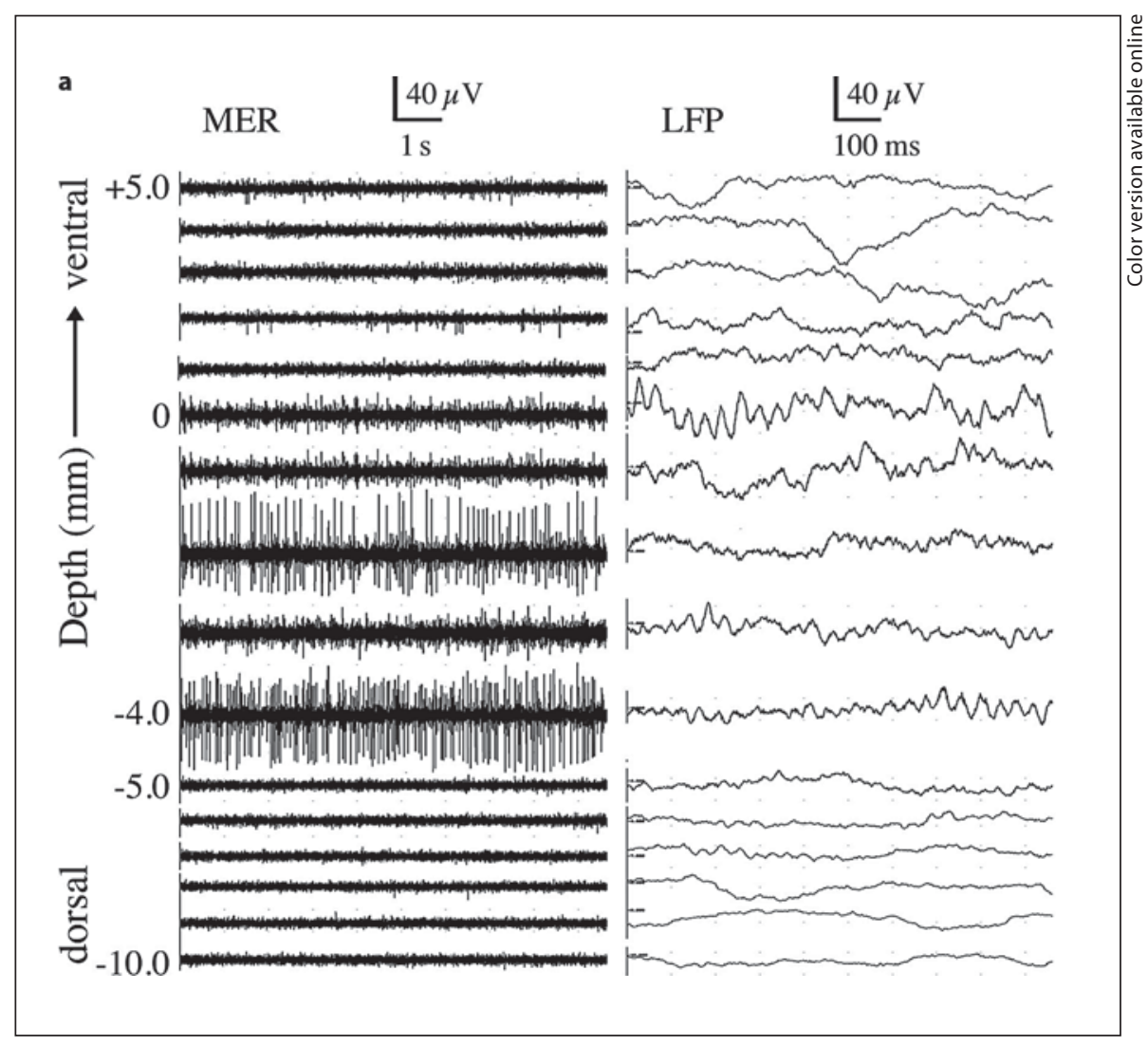

Fig. 2. An example of PSD of local field potential around the STN recorded by DBS electrode. a Raw data of MER and LFP obtained from a 65-year-old patient. LFP was obtained during advancing DBS lead after MER. b The relationship between the depth and PSD. The image was constructed by offline analysis using MATLAB (The MathWorks, Inc., Mass., USA). The $\mathrm{x}$-axis indicates the frequency in Hertz and the $y$-axis indicates the depth of the bipolar center of the lowermost contacts of the DBS electrode, advancing from $15 \mathrm{~mm}$ dorsal to $5 \mathrm{~mm}$ ventral to the tentative target (depth $0 \mathrm{~mm}$ ) through the STN. A color bar on the right represents power in square microvolt/Hertz. 


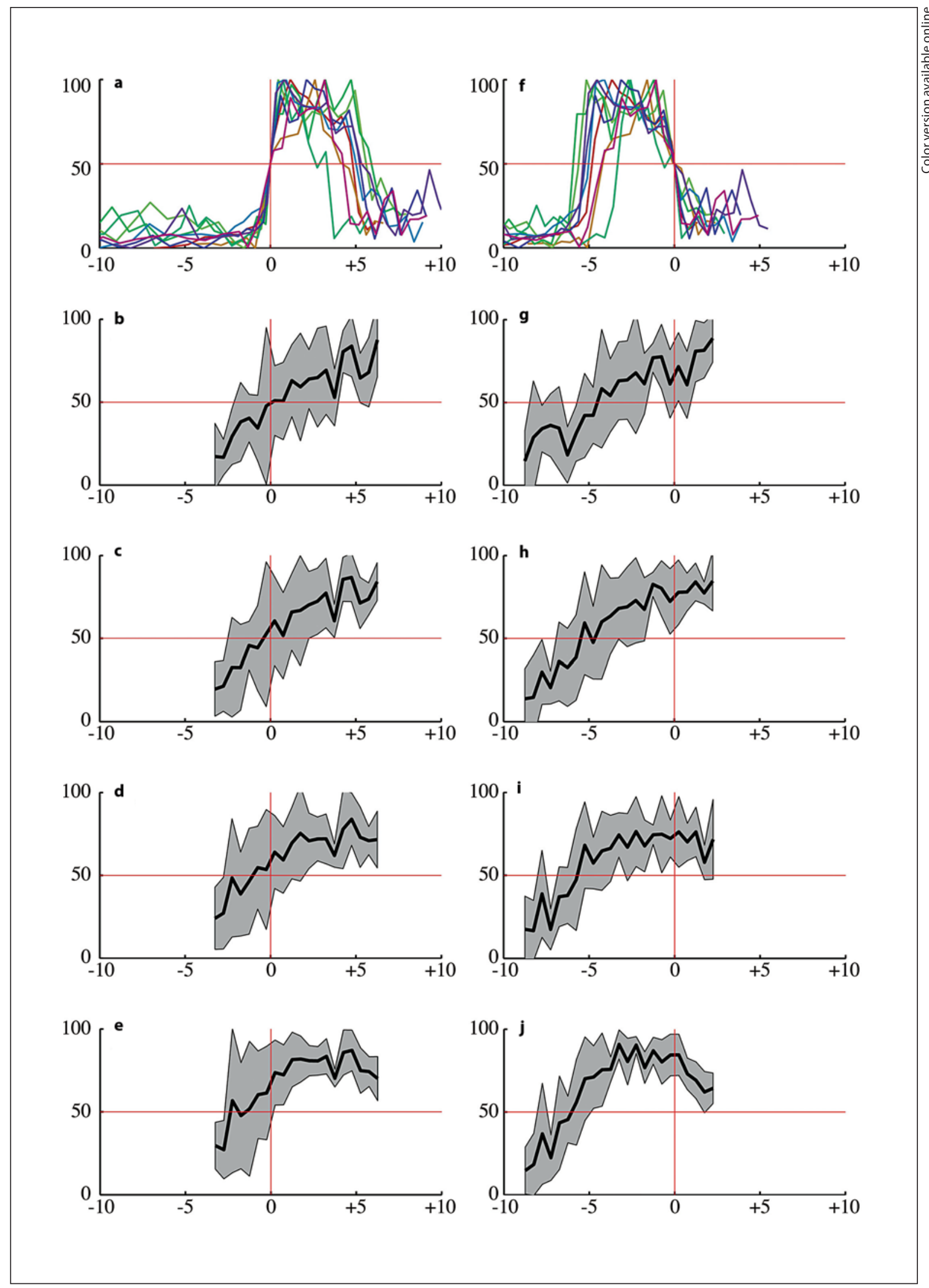


cells were distributed in the dorsal STN [5]. Since these LFP analyses were performed by tetrode or multiple microelectrodes, the LFP signals were interpreted to originate from an area narrow enough to localize the borders of the STN. However, the DBS electrode in the present study may have received the oscillatory LFP extensively from the surrounding structures other than the STN, especially the SNr. Therefore, the $\beta$-power recorded by DBS electrode did not show any clear distribution dominant in the dorsal area of the STN as explained in previous physiological studies [3, 5] and anatomical research [17]. Moreover, the increase in LFP power was not specific to $\beta$-band frequency in the present study, which did not agree with the microelectrode study by Trottenberg et al. [4].

Furthermore, the thickness of the DBS electrode (1.27$\mathrm{mm}$ diameter with a blunt tip) will cause a microlesion or microedema during insertion which is usually recognized as a transitory improvement of parkinsonian motor symptom (microlesioning effect) [18]. So, it is probable that the low-frequency oscillatory activity might have been considerably influenced by the intraoperative microlesioning effect of the DBS lead itself, as compared with MER [3-5] or the DBS electrode recording several days postoperatively [9].

STN DBS is now accepted as a powerful surgical approach for advanced PD with intractable motor fluctuation [19]. An image-guided anatomical targeting and physiological verification of the nucleus with MER is crucial to achieve an accurate implantation of a DBS electrode in the sensorimotor part (dorsolateral region) of the STN, which is commonly assumed to be the most directly related to the development of cardinal motor fluctua- tion in PD and therefore the best surgical target for DBS $[20,21]$. In particular, the physiologically determined width of the STN larger than $4 \mathrm{~mm}$ is considered to be one of the reliable determinants of an effective trajectory for STN DBS $[10,11,14]$. The preliminary results from this study suggest that the dorsal border of the STN can be estimated by the increase in low-frequency powers recorded with the DBS electrode and a decline in high $\beta$ power may indicate the ventral border. However, further physiological techniques for detecting the range of the sensorimotor area of the STN would be needed, such as synchronization or desynchronization of neuronal activity in response to motor task or stimulation [15], to replace the role of MER by DBS electrode recording.

\section{Conclusion}

In addition to MER study, a spectral power analysis of LFP recorded by DBS electrode is useful for the final confirmation of the physiological borders of the STN in DBS implantation surgery. Further physiological studies and online data analyses are required to define the exact borders of the sensorimotor STN, if subthalamic LFPs substitute the role of MER.

\section{Acknowledgement}

We thank Ms. Nancy Venarske and Dr. Daniel Venarske for their kind assistance in preparing this manuscript. This study was supported by Aihara Complexity Modelling Project, ERATO, Japan Science and Technology Agency.

\section{References}

Fig. 3. Summary of offline analysis of the background MER activity and low-frequency LFP power around the STN. a The background activities in MER from 9 STNs were realigned in reference to the dorsal border $(0 \mathrm{~mm})$ of the STN, which was defined as a point of $50 \%$ increase of the maximum SD from the baseline. f Similarly, the background activities in MER from 9 STNs were realigned in reference to the ventral border $(0 \mathrm{~mm})$ of the STN, which was defined as a point of $50 \%$ decrease from the maximum value. $\mathbf{b}-\mathbf{e}, \mathbf{g}-\mathbf{j}$ The relationships between the depth along the trajectory and LFP power of low-frequency band. The data were expressed as means (thick line) \pm SD (grey zone). Changes in LFP powers were realigned in reference to the dorsal border $(\mathbf{b}-\mathbf{e})$ and the ventral border $(\mathbf{g}-\mathbf{j})$. b, $\mathbf{g} \theta$-Band, $4-7 \mathrm{~Hz}$. c, $\mathbf{h} \alpha$-Band, $8-13$ Hz. d, i Low $\beta$-band, 14-20 Hz. e, j High $\beta$-band, 21-35 Hz. The negative and positive values in depth mean dorsal and ventral to the border $(0 \mathrm{~mm})$, respectively.

Subthalamic Field Potential by DBS Lead P: Reduction in subthalamic $8-35 \mathrm{~Hz}$ oscillatory activity correlates with clinical improvement in Parkinson's disease. Eur J Neurosci 2006;23:1956-1960.

2 Brown P, Williams D: Basal ganglia local field potential activity: character and functional significance in the human. Clin Neurophysiol 2005; 116:2510-2519.

3 Kühn AA, Trottenberg T, Kivi A, Kupsch A, Schneider GH, Brown P: The relationship between local field potential and neuronal discharge in the subthalamic nucleus of patients with Parkinson's disease. Exp Neurol 2005; 194:212-220.

4 Trottenberg T, Kupsch A, Schneider GH, Brown P, Kühn AA: Frequency-dependent distribution of local field potential activity within the subthalamic nucleus in Parkinson's disease. Exp Neurol 2007;205:287-291. 
5 Weinberger M, Mahant N, Hutchison WD, Lozano AM, Moro E, Hodaie M, Lang AE, Dostrovsky JO: Beta oscillatory activity in the subthalamic nucleus and its relation to dopaminergic response in Parkinson's disease. J Neurophysiol 2006;96:3248-3256.

6 Chen CC, Pogosyan A, Zrinzo LU, Tisch S, Limousin P, Ashkan K, Yousry T, Hariz MI, Brown P: Intra-operative recordings of local field potentials can help localize the subthalamic nucleus in Parkinson's disease surgery. Exp Neurol 2006;198:214-221.

7 Foffani G, Ardolino G, Egidi M, Caputo E, Bossi B, Priori A: Subthalamic oscillatory activities at beta or higher frequency do not change after high-frequency DBS in Parkinson's disease. Brain Res Bull 2006;69:123130.

8 Liu X, Ford-Dunn HL, Hayward GN, Nandi D, Miall RC, Aziz TZ, Stein JF: The oscillatory activity in the parkinsonian subthalamic nucleus investigated using the macro-electrodes for deep brain stimulation. Clin Neurophysiol 2002;113:1667-1672.

9 Kempf F, Kühn AA, Kupsch A, Brucke C, Weise L, Schneider GH, Brown P: Premovement activities in the subthalamic area of patients with Parkinson's disease and their dependence on task. Eur J Neurosci 2007;25: 3137-3145.
10 Saint-Cyr JA, Hoque T, Pereira LC, Dostrovsky JO, Hutchison WD, Mikulis DJ, Abosch A, Sime E, Lang AE, Lozano AM: Localization of clinically effective stimulating electrodes in the human subthalamic nucleus on magnetic resonance imaging. J Neurosurg 2002;97:1152-1166.

11 Sterio D, Zonenshayn M, Mogilner AY, Rezai AR, Kiprovski K, Kelly PJ, Beric A: Neurophysiological refinement of subthalamic nucleus targeting. Neurosurgery 2002;50:5867; discussion 67-59.

12 Miyagi Y, Shima F, Sasaki T: Brain shift: an error factor during implantation of deep brain stimulation electrodes. J Neurosurg 2007;107:989-997.

13 Benazzouz A, Breit S, Koudsie A, Pollak P, Krack P, Benabid AL: Intraoperative microrecordings of the subthalamic nucleus in Parkinson's disease. Mov Disord 2002; 17(suppl 3):S145-S149.

14 McClelland S 3rd, Kim B, Winfield LM, Ford B, Edwards TA, Pullman SL, Yu Q, McKhann GM 2nd, Goodman RR: Microelectrode-recording-determined subthalamic nucleus length not predictive of stimulation-induced side effects. Neurosurg Focus 2005;19:E13.

15 Kühn AA, Williams D, Kupsch A, Limousin P, Hariz M, Schneider GH, Yarrow K, Brown P: Event-related beta desynchronization in human subthalamic nucleus correlates with motor performance. Brain 2004;127:735746.
16 Welch PD: The use of fast Fourier transform for the estimation of power spectra: a method based on time averaging over short, modified periodograms. IEEE Trans Audio Electroacoust 1967;15:70-73.

17 Lévesque J-C, Parent A: GABAergic interneurons in human subthalamic nucleus. Mov Disord 2005;20:574-584.

18 Deuschl G, Herzog J, Kleiner-Fisman G, Kubu C, Lozano AM, Lyons KE, RodriguezOroz MC, Tamma F, Tröster AI, Vitek JL, Volkmann J, Voon V: Deep brain stimulation: postoperative issues. Mov Disord 2006; 21(suppl 14):S219-S237.

19 Krack P, Batir A, Van Blercom N, Chabardes S, Fraix V, Ardouin C, Koudsie A, Limousin PD, Benazzouz A, LeBas JF, Benabid AL, Pollak P: Five-year follow-up of bilateral stimulation of the subthalamic nucleus in advanced Parkinson's disease. N Engl J Med 2003;349:1925-1934

20 Godinho F, Thobois S, Magnin M, Guenot M, Polo G, Benatru I, Xie J, Salvetti A, Garcia-Larrea L, Broussolle E, Mertens P: Subthalamic nucleus stimulation in Parkinson's disease: anatomical and electrophysiological localization of active contacts. J Neurol 2006; 253:1347-1355.

21 Yokoyama T, Sugiyama K, Nishizawa S, Tanaka T, Yokota N, Ohta S, Uemura K: Neural activity of the subthalamic nucleus in Parkinson's disease patients. Acta Neurochir (Wien) 1998;140:1287-1290. 